\title{
BMJ Open Do patterns of mental healthcare predict treatment failure in young people with schizophrenia? Evidence from an Italian population-based cohort study
}

\author{
Giovanni Corrao, ${ }^{1}$ Davide Soranna, ${ }^{1}$ Luca Merlino, ${ }^{2}$ Emiliano Monzani, ${ }^{3}$ \\ Caterina Viganò, ${ }^{4}$ Antonio Lora ${ }^{5}$
}

To cite: Corrao G, Soranna D, Merlino L, et al. Do patterns of mental healthcare predict treatment failure in young people with schizophrenia? Evidence from an Italian population-based cohort study. BMJ Open 2015;5: e007140. doi:10.1136/ bmjopen-2014-007140

- Prepublication history for this paper is available online. To view these files please visit the journal online (http://dx.doi.org/10.1136/ bmjopen-2014-007140).

Received 10 November 2014 Revised 27 March 2015 Accepted 8 April 2015

CrossMark

For numbered affiliations see end of article.

Correspondence to Professor Giovanni Corrao; giovanni.corrao@unimib.it

\section{ABSTRACT}

Objective: Little is known about the practice of predicting community-based care effectiveness of patients affected by schizophrenic disorders. We assessed predictors of treatment failure in a large sample of young people affected by schizophrenia. Methods: A cohort of 556 patients aged 18-35 years who were originally diagnosed with schizophrenia during 2005-2009 in a Mental Health Service (MHS) of the Italian Lombardy Region was identified. Intensity of mental healthcare received during the first year after index visit (exposure) was measured by patients' regularity in MHS attendance and the length of time covered with antipsychotic drug therapy. Patients were followed from index visit until 2012 for identifying hospital admission for mental disorder (outcome). A proportional hazards model was fitted to estimate the HR and $95 \%$ Cls for the exposure-outcome association, after adjusting for several covariates. A set of sensitivity analyses were performed in order to account for sources of systematic uncertainty.

Results: During follow-up, 144 cohort members experienced the outcome. Compared with patients on low coverage with antipsychotic drugs ( $\leq 4$ months), those on intermediate (5-8 months) and high ( $\geq 9$ months) coverage, had HRs $(95 \% \mathrm{Cl}$ ) of 0.94 ( 0.64 to 1.40 ) and 0.69 (0.48 to 0.98 ), respectively. There was no evidence that regular attendance at the MHS affected the outcome.

Conclusions: Patients in the early phase of schizophrenia and their families should be cautioned about the possible consequences of poor antipsychotic adherence. Physicians and decision makers should increase their contribution towards improving mental healthcare.

\section{INTRODUCTION}

The fifth edition of the Diagnostic and Statistical Manual of Mental Disorders defines schizophrenia as a syndrome characterised by a long duration and high relapse rate, with abnormalities in one or more of the following five domains: delusions, hallucinations, disorganised thinking (speech),
Strengths and limitations of this study

- The investigation is based on data from a large unselected population that were available since practically all citizens in Italy are covered by a cost-free healthcare system.

- Our data reflect routine clinical practice and are not affected by selective participation and recall bias.

- Patients were identified from the point of the initial visit with the mental health service in which diagnosis of schizophrenia was made, and the complete sequence of public or accredited healthcare facilities, including mental healthcare and other services, was known.

- Evaluation of antipsychotic drug coverage was based on pharmacy-dispensing information. This method assumes that the proportion of days covered by a prescription corresponds to the proportion of days of medication use.

- Notwithstanding the large sample size, our study was not sufficiently powered to investigate the effect of relatively rare exposures.

grossly disorganised or abnormal motor behaviour, including catatonia and negative symptoms, and sometimes mood problems. ${ }^{1}$ The onset of symptoms typically occurs in adolescence and young adulthood, with a worldwide estimate of lifetime prevalence and incidence of 1.4-4.6 and 0.16-0.42 per 1000 persons annually, respectively. ${ }^{2} 3$ A recent systematic review indicated that patients diagnosed with this disorder have a shorter lifespan than the average general population. Furthermore, they have an increased risk of suicide and physical impairment (eg, limited exercise, poor diet and obesity), and reduced access to medical treatment and healthcare services. ${ }^{4}$

Antipsychotic medications have been recommended as the mainstream of medical treatment for nearly all patients with 
schizophrenia, to provide them with a safe and therapeutic environment, and effective symptom control. ${ }^{5}$ In the last few decades, different approaches to psychosocial interventions (eg, psychotherapy, family therapy, psychoeducation and cognitive-behavioural therapy) have shown to be able to bear positive patient outcomes, admitted that they are used as an adjunct to antipsychotics. $^{6-13}$ Although their comparative efficacy has been increasingly evaluated in various clinical trials, ${ }^{7} 91112$ it should be emphasised that patients enrolled in these trials were likely quite motivated, as they were consenting to intense monitoring in a structured research programme, and likely adhered to, or were controlled for adherence, with clinical recommendations. Unfortunately, however, how these intervention programmes perform in psychiatrically ill patients treated in real-world, naturalistic settings, is little known.

Mental health services (MHS) play a central role in the treatment of people with schizophrenic disorders, as they act both as direct providers of care and as supporters of primary care practitioners. ${ }^{14}$ In accordance with the Italian model of MHS, as well as with that of most high-income countries, a complex network of different types of community mental health teams, and a range of treatment, rehabilitation, employment and residential care facilities, currently operate in the community. ${ }^{15}$ To date, however, little is known about the practice and patient characteristics that predict effectiveness of community-based care patients affected by schizophrenic disorders. This paper is designed to address this need. Our purpose is to assess predictors of treatment failure in a large and unselected sample of young people affected by schizophrenia.

\section{METHODS}

Departments of mental health in Lombardy

Lombardy, the largest and the most affluent region in Italy with, in 2009, a population of about 9700000 , lies in the northernmost part of the country. In Lombardy, the public Department of Mental Health (DMH) provides mental healthcare through a network of community services, ranging from acute emergency treatment to long-term rehabilitation; it therefore includes one or more of all the following facilities: community Mental Health Centres, General Hospital Psychiatric Wards (GHPW), Day Care Centres, community Residential Facilities (RF). The public DMH is an administrative, financial and organisational entity, and is fully responsible for the network of community mental health facilities in the catchment area, including GHPWs and public RFs. It has full governance of all the available facilities in the Department, and also authorises and controls the admission of DMH patients to private, licensed facilities, such as private RFs. ${ }^{16}$

\section{Healthcare utilisation databases of Lombardy}

In Italy, the population is covered by the National Health Service (NHS), and in Lombardy, its management has been associated with an automated system of databases since 1997, which collect a variety of information concerning services provided to beneficiaries of NHS. Information includes data on patients attending public DMH, for example, demographics, International Classification of Diseases, 10th edition (ICD-10) diagnoses, treatments such as outpatient and home visits, day treatment attendance and admittance to RFs. Besides the specific system for monitoring the use of MHS, other automated systems for monitoring healthcare utilisation include: databases on diagnosis at discharge from public or private hospitals; outpatient drug prescriptions reimbursed by the NHS: drug prescriptions administered directly in the outpatient setting and day hospital; and outpatient services, such as visits and diagnostic tests, respectively, performed in specialist ambulatories and laboratories accredited by the NHS. For each patient, we linked the above databases via a single identification code. In order to preserve privacy, each identification code was automatically converted to an anonymous code. The inverse process was prevented by deletion of the conversion table.

Through this record linkage process we were able to mark out the complete care pathway of all the citizens of Lombardy who are beneficiaries of NHS, comprising practically all residents in the region, through a long period of observation. This offers the opportunity to design investigations including very large unselected populations, and to generate real-world evidence on several fields of healthcare, including mental health. ${ }^{17} 18$

\section{Cohort selection and follow-up}

The target population consisted of all beneficiaries of the NHS resident in Lombardy and aged $18-35$ years. According to the 2011 Italian Census, this population amounted to 1893313 individuals. Of these, we identified patients who, during 1 January 2005 to 31 December 2009, had at least one contact with an MHS accredited by the NHS, and who received, on that occasion, the diagnosis of schizophrenia spectrum disorder (ICD-10 codes 2X.XX). The date of the first visit during the considered period was recorded as the index visit.

With the aim of favouring the inclusion of only newly treated individuals, patients who within 2 years prior to the index visit experienced at least one hospital admission for mental disorder (ICD-9 290-319), had at least one contact with an MHS, or who simply received a prescription for an antipsychotic agent, were excluded. Patients who, during the first year after index visit, did not receive antipsychotic medicaments, were also excluded, based on the assumption that in these patients, continuous drug treatment might not have been indicated. Finally, patients who did not reach at least 1 year of follow-up were excluded, to ensure at least 1 year of potential exposure to the care of interest. The remaining patients represented the study cohort.

Each member of the cohort accumulated person-years of follow-up from the index visit until the earliest among 
the dates of outcome onset (ie, hospital admission for mental disorder) or censoring (ie, death from any cause, emigration, or 31 December 2012).

\section{Characterising cohort members}

For each cohort member, data included selected tracts detected at the index visit, such as gender, age, social tracts and physical comorbidities. The latter were measured by means of the Charlson comorbidity index score, ${ }^{19}$ which was calculated via the diagnostic information available from inpatient charts in the 2 years prior, and 1 year after, the index visit; patients were categorised as either having or not having chronic comorbidities. Finally, cotreatments with antidepressive and mood stabilisers during the first year after the index visit were considered.

\section{Measuring mental healthcare}

All contacts with an MHS experienced by the cohort members during the first year after the index visit were identified. Attendance was considered regular if the time span between two consecutive visits was 60 days or shorter, or as discontinued, otherwise. Patients were classified into two mutually exclusive categories of those who had regular attendance with the MHS (ie, if they did not experience any episode of discontinuation through the first year after the index visit), or were discontinuers otherwise.

Patients were, furthermore, classified according to if they received at least three individual, familiar or group sessions of psychotherapy, family psychoeducational activities or other supports (eg, social, expressive, practical-manual, physical activities, and support in independent living and in employment).

Data were also regarded in relation to the professionals who supplied care, distinguishing between patients who were seen only by mental health staff (psychiatrists and psychologists), or also by other professionals (educators, social workers, etc).

Coverage with antipsychotic drug therapy was measured by means of the cumulative number of days during which the medication was available. Patients were, accordingly, categorised as having at least 4 months, from 5 to 8 months, and more than 9 months of drug coverage.

\section{Data analysis}

The Kaplan-Meier estimator of the cumulative proportion of patients experiencing the outcome was built for the entire cohort, as well as according to selected subgroups. The log-rank test was used to rate between group differences. The Cox proportional hazard regression model was fitted to estimate the HR and its $95 \%$ CI for the association between the above listed covariates, and time of outcome onset. Trends in HRs were tested, when feasible, according to the statistical significance of the regression coefficient of the recoded variables obtained by scoring the corresponding categories.

\section{Sensitivity analyses}

Because of the arbitrariness of taking a 60-day time span to distinguish regular and discontinuing attendance, and to account for at least three psychotherapy sessions to identify patients treated, data were reanalysed according to different criteria, such as 30-day and 90-day time spans, and one or two sessions of psychotherapy.

The robustness of our findings with regard to potential bias introduced by unmeasured confounders was investigated by using the rule-out approach described by Schneeweiss. ${ }^{20}$ Let $\mathrm{E}$ be the exposure of interest (eg, the regular attendance to the MHS contrasted with discontinuation), $\mathrm{O}$ the outcome (ie, hospital admission for mental disorder) and $\mathrm{C}$ a hypothetical unmeasured confounder (eg, severity of schizophrenia at diagnosis). In applying the rule-out method, we allowed C: (1) to be present in the study population with a prevalence of $50 \%$ (eg, one half of included patients had symptoms of severe schizophrenia at index visit); (2) to be associated with $\mathrm{O}$ with risk ratio varying from 1 to 10 (ie, severe schizophrenics may experience the outcome up to 10-fold more than patients with less severe symptoms) and (3) to be associated with $\mathrm{E}$ with $\mathrm{OR}$ varying from 1 to 10 (ie, severe schizophrenics may regularly attend the service up to 10-fold more than others). In its original formulation, the rule-out approach aims to detect the extension of confounding required to fully account for the observed exposure-outcome association, so as to drag the observed point estimate towards that expected under the null hypothesis. In our application, we extended the use of the rule-out approach in the situations where the observed association did not reach statistical significance, and the interest was to detect the extension of confounding required to make statistically significant the exposure-outcome association. With this aim, we conducted the analysis for the value of the observed higher $95 \%$ confidence limit to determine the constellations in which the $95 \%$ CI would not cross the expected value under the null hypothesis.

\section{RESULTS}

The distribution of the exclusion criteria is shown in figure 1. The 556 patients who met inclusion criteria accumulated 2619 person-years of observation, with an average per-patient follow-up of about 4.7 years. During this period, 144 patients belonging to the cohort members experienced the outcome.

Table 1 provides some characteristics of the included cohort members. At the index visit, almost one half of the patients were aged $30-35$ years, and $68 \%$ of them were men. Most patients had poor education, were never married and/or lived as a family and almost $60 \%$ were unemployed. Only two patients showed at least one sign of chronic comorbidity. More than one half of the patients were co-treated with antidepressive agents, and one in seven patients was co-treated with mood stabiliser agents. Among the considered covariates were evidence 
Figure 1 Flow chart of inclusion and exclusion criteria. $\mathrm{DMH}$, Department of Mental Health; Lombardy Region, Italy, 20052012

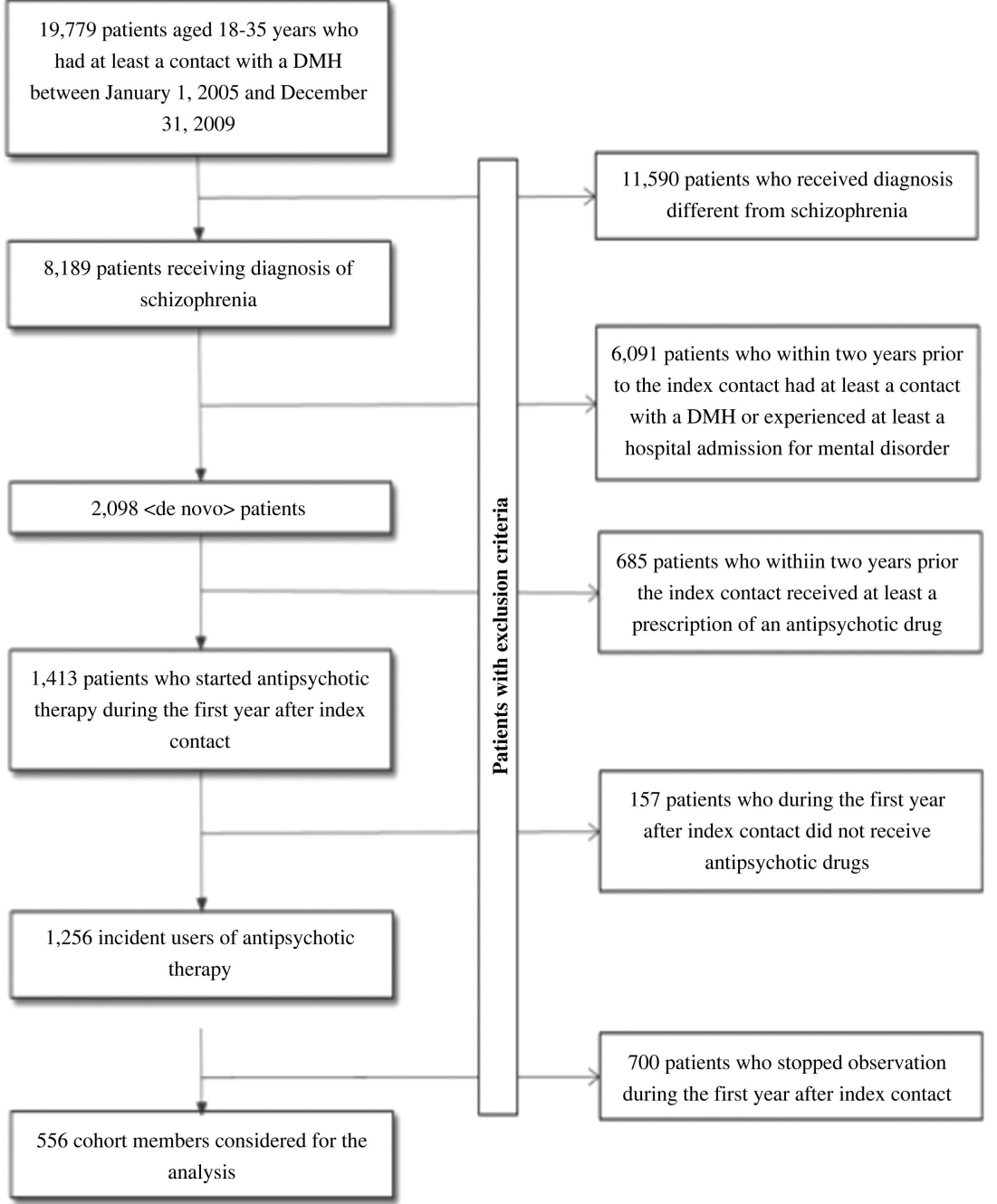

of increased outcome risk issued for patients with poor education, currently unemployed, and those who did not receive antidepressive agents.

As far as mental healthcare provided to cohort members during the first year after index visit is concerned, table 2 shows that, although only one in five patients had good coverage with antipsychotic drug therapy, significant reduction of the outcome risk was observed for these patients. The inverse coverageoutcome relationship is depicted in figure 2. In particular, the cumulative proportion of patients experiencing the outcome decreased from $34 \%$ to $32 \%$ and $23 \%$ for patients with low, intermediate and high drug coverage, respectively.

Table 2 shows that most patients received interventions delivered by professionals other than psychiatrists and psychologists; $12 \%$ received at least three sessions of psychotherapy, and one-quarter of patients had regular MHS attendance without any episode of discontinuation during the first year after the index visit. There was no evidence that type of treatment, role of caregivers, or regular attendance with MHS, affected the outcome risk.

As expected, the proportion of regularly attending patients, as well as that of adequately treated patients, decreases as less permissive criteria were adopted. Figure 3 shows that regulars accounted for only $5 \%$ by allowing 30 days between two consecutive visits, while $42 \%$ of patients had 'regular' contacts at least every 90 days. Among the 124 patients who received a first psychotherapy session, only $66(53 \%)$ reached at least three sessions. It should be noted, however, that the lack of evidence of a protective effect of care was provided by territorial services even by varying the classification criteria.

The results of the residual confounding analysis obtained by means of the rule-out approach are presented in figure 4 . If we assume that patients exposed to the unmeasured confounder have a fourfold increased outcome risk than those unexposed (ie, severe schizophrenics experience the outcome fourfold more than others), then patients exposed to the confounder would increase their exposure of 5.3-fold or more (ie, severe schizophrenics would regularly attend the service with odds more than fivefold higher than others) to influence the effect of regular attendance to be significantly protective on the outcome risk. On the other hand, if we assume that patients exposed to the confounder have even higher outcome risk (eg, severe schizophrenics 
Table 1 Selected tracts of the 556 included patients with diagnosis of schizophrenia and their relationship with the long-term risk of hospital admission for mental disorders (Lombardy Region, Italy, 2005-2012)

\begin{tabular}{|c|c|c|c|}
\hline & N (\%) & $\begin{array}{l}\text { Rough HR H } \\
(95 \% \text { Cl) }\end{array}$ & $\begin{array}{l}\text { Adjusted HR* } \\
(95 \% \mathrm{Cl})\end{array}$ \\
\hline \multicolumn{4}{|l|}{ Demographics $\dagger$} \\
\hline Male gender & $80(68)$ & $1.05(0.74$ to 1.49$)$ & 1.04 (0.72 to 1.49$)$ \\
\hline \multicolumn{4}{|l|}{ Age (years) } \\
\hline $18-23$ & $144(26)$ & 1.00 (reference) & 1.00 (reference) \\
\hline $24-29$ & $160(29)$ & $0.72(0.46$ to 1.11$)$ & 0.75 (0.46 to 1.17$)$ \\
\hline $30-35$ & $252(45)$ & $0.83(0.56$ to 1.22$)$ & 0.89 (0.60 to 1.33$)$ \\
\hline$p_{\text {trend }} \ddagger$ & & 0.269 & 0.343 \\
\hline \multicolumn{4}{|l|}{ Social tracts $†$} \\
\hline \multicolumn{4}{|l|}{ Education (years) } \\
\hline$\leq 8$ & $323(58)$ & 1.00 (reference) & 1.00 (reference) \\
\hline $9-14$ & $195(35)$ & $0.72(0.05$ to 1.03$)$ & 0.80 (0.55 to 1.16$)$ \\
\hline$>14$ & $38(7)$ & $0.49(0.22$ to 1.12$)$ & 0.51 (0.26 to 0.99$)$ \\
\hline$p_{\text {trend }} \S$ & & 0.020 & 0.046 \\
\hline \multicolumn{4}{|l|}{ Marital status } \\
\hline Married & $94(17)$ & 1.00 (reference) & 1.00 (reference) \\
\hline Never married & $462(83)$ & $0.95(0.62$ to 1.46$)$ & 0.88 (0.55 to 1.42$)$ \\
\hline \multicolumn{4}{|l|}{ Living arrangements } \\
\hline Alone & $34(6)$ & 1.00 (reference) & 1.00 (reference) \\
\hline Family & $513(92)$ & $1.04(0.51$ to 2.12$)$ & 0.70 (0.49 to 1.00$)$ \\
\hline Community & $9(2)$ & & \\
\hline \multicolumn{4}{|l|}{ Employment } \\
\hline Currently unemployed & $324(58)$ & 1.00 (reference) & 1.00 (reference) \\
\hline Currently employed & $232(42)$ & 0.65 (0.46 to 0.91$)$ & 0.74 (0.55 to 0.99$)$ \\
\hline \multicolumn{4}{|c|}{ Cotreatments and comorbidities } \\
\hline \multicolumn{4}{|c|}{ Physical comorbidities』 } \\
\hline None & 554 (99) & - & - \\
\hline One or more & $2(1)$ & - & - \\
\hline \multicolumn{4}{|l|}{ Cotreatments $\ddagger$} \\
\hline Antidepressive & $274(49)$ & 0.67 (0.48 to 0.93$)$ & 0.66 (0.47 to 0.93 ) \\
\hline Mood stabilisers & $84(15)$ & 1.41 (0.93 to 2.14$)$ & 1.51 (0.98 to 2.33$)$ \\
\hline \multicolumn{4}{|c|}{$\begin{array}{l}\text { Estimates were rough and mutually adjusted for covariates listed in tables } 1 \text { and } 2 \text {. } \\
\text { *HR (and } 95 \% \text { CI) for the risk of hospital admission for mental disorders, according to a Cox proportional hazard model. } \\
\text { tAt baseline (index visit). } \\
\text { fDuring the first year after index visit. } \\
\text { \$p Value for the trend in the risk of outcome as the category of the corresponding variable increases. } \\
\text { TAccording to diagnostic information available from inpatient charts in the } 2 \text { years prior and } 1 \text { vear after the index visit. }\end{array}$} \\
\hline
\end{tabular}

experience the outcome 10 -fold more than others), then a lower discrepancy of the exposure of interest is required to admit protection (ie, severe schizophrenics would regularly attend the service more than threefold higher than others).

\section{DISCUSSION}

Our population-based cohort study shows that mental healthcare provided to young schizophrenic patients is frequently abandoned and/or is not adequately followed up since the first year after diagnosis. Consistently, and with an impressive amount of literature, ${ }^{21}$ this was observed with regard to antipsychotic drug therapy, since only one in five patients had good coverage with antipsychotic drug therapy. Furthermore, only 1 patient in 20 had monthly attendance of a territorial MHS, and $58 \%$ of schizophrenics had attended the service less than once every 3 months.
An important finding of our study is that, in the realworld setting, the longer the coverage with antipsychotic drug therapy during the first year after diagnosis of schizophrenia, the greater the reduction in the risk of long-term hospital admission for mental disorder. Thus, our findings suggest that treatment with antipsychotics may favourably affect the risk of hospitalisation, a conclusion that expands, to a large unselected population, the conclusions of several observational studies, ${ }^{22-25}$ and a recent systematic review of randomised clinical trials. ${ }^{26}$ The poor compliance with drug therapy in everyday clinical practice, together with the observed strength of the association between drug coverage and outcome, given a $21 \%$ attributable fraction, which is more than one-fifth of outcome currently occurring among schizophrenics who received antipsychotic drug therapy at baseline, may be prevented if all those patients had good coverage with therapy. Hence, among the investigated factors, poor coverage 


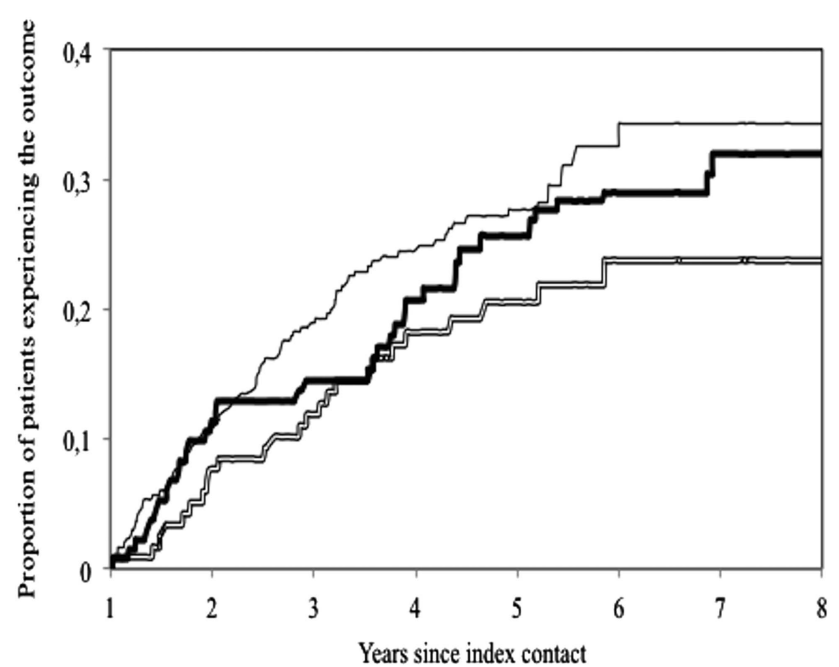

Coverage with antipsychotic drug therapy

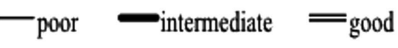

Figure 2 Cumulative proportion of patients experiencing hospitalisation for mental disorders, according to their coverage with antipsychotic drug therapy during the first year since index visit. Lombardy Region, Italy, 2005-2012.

with antipsychotic drugs appears the more important predictor.

Another important finding of our study is that, unexpectedly, regular attendance of a territorial MHS for receiving psychotherapy, psychoeducational activities, social supports and multidisciplinary care, did not show evidence of exerting a protective effect on the risk of hospitalisation in our setting. Although it is difficult to draw consistent conclusions from the existing literature on the efficacy of psychological interventions in the therapy of schizophrenic patients, there is, however, sound evidence for the efficacy of specific psychological approaches, that is, social skills training, cognitive remediation, psychoeducational interventions with families and relatives, and cognitive behavioural therapy of psychotic symptoms. ${ }^{11}$ This suggested their implementation into routine care according to several national guidelines. ${ }^{27}$ However, the main open question remains the generalisability of randomised control trial findings to routine care. Our study suggests that the gap between what is known from clinical efficacy research and the systematic community translation of mental healthcare programmes is still dramatically wide, at least in the investigated setting.

Other findings of our study deserve to be mentioned. First, we observed that almost one half of the included patients were aged 30-35 years, that is, they were much older than expected for a group experiencing a first episode of psychosis. This reveals that accessibility to public MHS should be improved for intercepting early onset of schizophrenic disorders. Second, among the considered social tracts, low level of education and unemployment were independent predictors of longterm risk of hospital admission for mental disorders, possibly due to the treatment delay, and then to the greater severity of illness at presentation, among people with low socioeconomic status. ${ }^{28}$ Finally, we observed that almost one half of the included patients co-utilised antidepressants, and that these patients were at lower risk of long-term mental disorder hospitalisation. This finding confirms recent evidence that antidepressants along with antipsychotics are more effective in treating the negative symptoms of schizophrenia than antipsychotics alone. ${ }^{29}$

The present study is unique in several respects. One, the investigation is based on data from a large unselected population, which was made possible since practically all citizens in Italy are covered by a cost-free healthcare system. Two, our data reflect routine clinical practice and are not affected by selective participation and recall bias. Three, patients were identified from the point of the initial visit with the MHS in which diagnosis of schizophrenia was made, and the complete sequence of public or accredited healthcare facilities, including mental healthcare and other services, was known. Four, accurate data are guaranteed from healthcare utilisation databases of Lombardy, as documented by several quality checks. ${ }^{30-32}$ Finally, a number of sensitivity analyses confirmed the robustness of our findings.

Our study may be limited by some issues. One, information about private mental health outpatient facilities is not available from our healthcare utilisation databases system. This involves the following systematic errors: (1) we selectively included patients who had at least a visit with a public structure; (2) we cannot exclude that some of the included patients already had visits with private structures, so that, despite our best efforts, some prevalent cases were selectively included and (3) the pathway of care that we were able to trace lacks for a part of clinical supplies. It should be emphasised, however, that the access to private facilities does not affect our ability to search out drug dispensations. In fact, according to the Italian health system organisation, free-of-charge drug prescriptions are, however, ensured (and then recorded in the healthcare utilisation database), even when they have been prescribed by a private physician. This can be explained because, among the investigated healthcare cases, only drug therapy showed evidence of effectiveness.

Two, evaluation of antipsychotic drug coverage was based on pharmacy-dispensing information. This method assumes that the proportion of days covered by a prescription corresponds to the proportion of days of medication use. Although data on dispensing history have shown to be consistent with other adherence measures, drug serum levels and clinical drug effects, ${ }^{33}$ it is likely that in a number of patients the prescribed drugs are not consumed. This implies that the use of medication dispensing as a measure of coverage remains a source of uncertainty of our estimates. It should be mentioned, however, that this source of misclassification likely leads to an underestimation of the strength of adherence-outcome association. ${ }^{34}$ 
Figure 3 Influence of criteria for identifying patients in regular attendance of the Mental Health Service and on psychotherapy on the HR for hospital admission for mental disorders. Lombardy Region, Italy, 2005-2012. Criteria concerning the time span within which two consecutive visits for allowing regular attendance (in box $A$, patients with regular attendance are contrasted with those who experience at least a discontinuing episode), and the cumulative number of psychotherapy sessions (in box $B$, patients with at least a given number of sessions are contrasted with those who experience fewer sessions). HR estimated according to Cox proportional hazard model. Estimates are adjusted for covariates listed in tables 1 and 2.

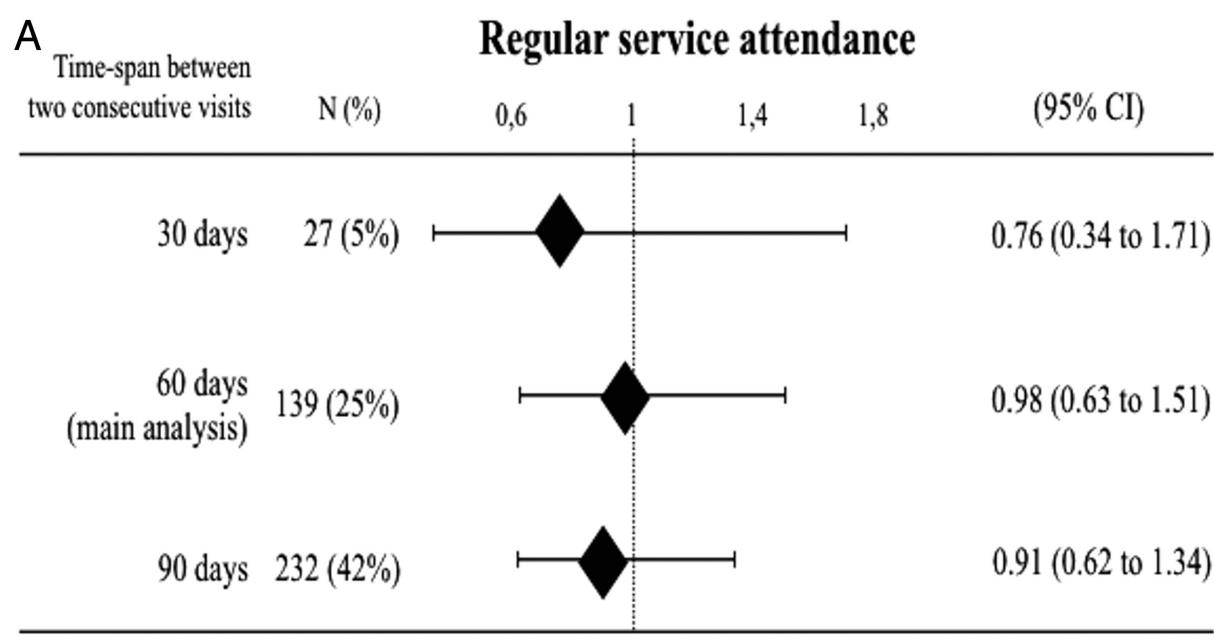

Better regular attendance

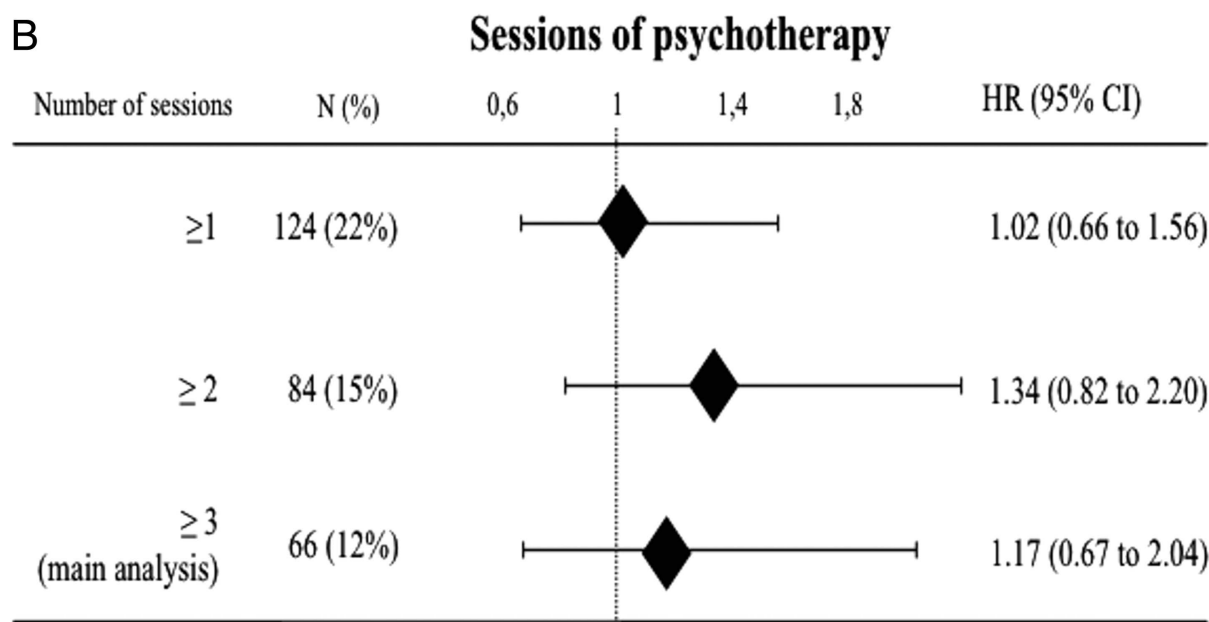

Better psychotherapy
Three, whether the observed findings are due to our inability to fully account for regular treatment to those patients at higher risk of clinical failure, is a relevant question in interpreting our findings. For example, it is likely that patients with severe schizophrenia at baseline are submitted to greater psychiatric attention than those with less severe symptoms, that is, more regular visits, greater care for therapeutic plan and longer drug coverage. Our study addressed confounding by means of the following insights. First, conventional adjustments for a number of available demographic, therapeutic and clinical characteristics, such as age, gender, social features and co-treatments, where most of them that may be considered proxies of disease severity, were performed. Second, we attempted to include patients at their first clinical manifestation of the disease. This was accomplished by excluding patients aged 35 years or over who had already received a diagnosis of schizophrenia, and those who had contacts with a department of mental health, experienced hospitalisations for mental disorder, or who had simply received antipsychotic drug dispensations. The exclusion of prevalent cases, as well as of prevalent drug users, is crucial for healthcare research, since the alternative consists in selectively including those patients who survived their disease status. $^{35}$ Third, we excluded schizophrenics who did not receive antipsychotic drug dispensations during the first year after the index visit. The latter exclusion criteria leading to research plans that we called 'only user design', 36 have been described as reducing the potential for confounding by indication. ${ }^{37}$ We suspect that, due to the nature of the precautions taken, confounding could have biased the effect of regular service attendance, rather than that of antipsychotic medicaments. Our sensitivity analysis accounting for unmeasured confounding, however, showed that considering severity of schizophrenia as the unmeasured factor, even assuming very high prevalence of severe schizophrenia at presentation $(50 \%)$, and that severe schizophrenics risk the outcome onset up to 10-fold more than others, a strong discrepancy in regularity of service attendance between severe and less severe schizophrenics would be needed for making the association of interest statistically significant. 


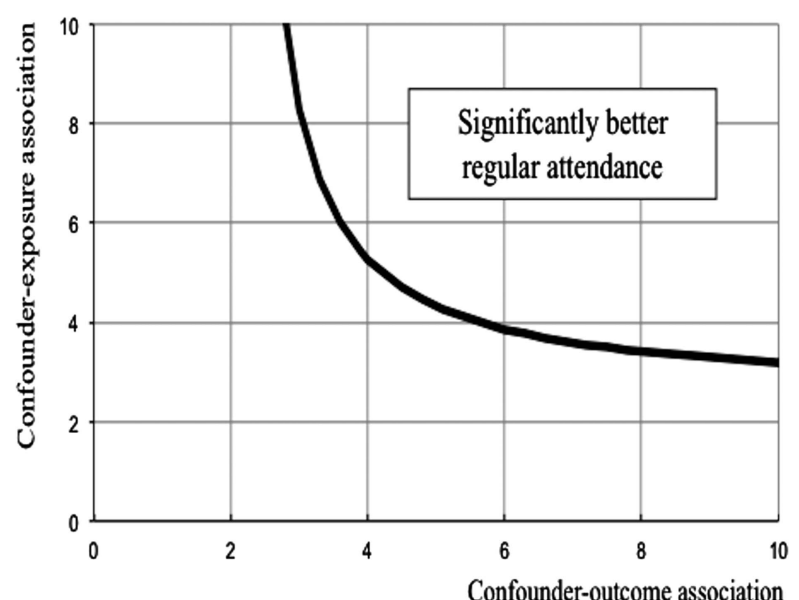

Figure 4 Modelled influence of a hypothetical confounder on the HR for hospital admission for mental disorders unaccounted for in the adjustments already performed in the main analysis. Lombardy Region, Italy, 2005-2012. The graph indicates what combinations of confounder-outcome and confounder-exposure would be required to make significantly protective the observed association between regular attendance of Mental Health Service and hospitalisation for mental disorders. For an explanation, see the 'Sensitivity analysis' in the subsection of the 'Methods' section.

Finally, although the sample size was large, our study was not sufficiently powered to investigate the effect of relatively rare exposures. For example, if we accept a permissive definition of regularity (eg, by requiring at least one contact every 3 months) then our study is able to generate significant evidence, admitted that discontinuers experience the outcome at least 1.8-fold more than regulars (with an error of the first type of $5 \%$ and a power of $80 \%$ ). On the other hand, we do not expect protection from such a permissive criterion. As the definition of regularity becomes less permissive, however, the observed number of regulars tends to decrease. For example, our study is able to generate significant evidence of outcome protection from monthly attendance, admitted that discontinuers experience the outcome at least fivefold more than regulars. This means that our study has limited chance of highlighting the impact of the proper use of the service, for the simple reason that proper use rarely occurs. Paradoxically, this source of weakness is due to the key message of our study, that is, the limited use of territorial service.

In conclusion, our data on care patterns provided to patients suffering from schizophrenic disorders in the real-world setting, show poor adherence with antipsychotic drug therapy, as well as with regular attendance of MHS. In addition, our study showed that good coverage with antipsychotic therapy within the first year after the diagnosis of schizophrenia reduces the longterm risk of hospital admission for mental disorders. Real-world psychosocial treatments, such as those provided at community level by MHS in Lombardy in the last decade, should be strengthened to be effective. The development in Lombardy in the last 4 years of more than 20 projects for the early treatment of psychosis is moving in this direction. It is thus important that mental health professionals and decision makers increase their contribution towards improving mental healthcare in clinical practice.

Table 2 Mental healthcare provided to the 556 included patients with diagnosis of schizophrenia and its relationship with the long-term risk of hospital admission for mental disorders (Lombardy Region, Italy, 2005-2012)

\begin{tabular}{|c|c|c|c|}
\hline & $\mathbf{N}(\%)$ & $\begin{array}{l}\text { Rough HR* } \\
(95 \% \mathrm{Cl})\end{array}$ & $\begin{array}{l}\text { Adjusted HR } \\
\text { (95\% Cl) }\end{array}$ \\
\hline \multicolumn{4}{|c|}{ Coverage with antipsychotic drug therapy (months)† } \\
\hline $1-4$ & $303(55)$ & 1.00 (reference) & 1.00 (reference) \\
\hline $5-8$ & $135(24)$ & $0.99(0.67$ to 1.45$)$ & $0.94(0.64$ to 1.40$)$ \\
\hline $9-12$ & $118(21)$ & 0.71 (0.51 to 0.99$)$ & $0.69(0.48$ to 0.98$)$ \\
\hline $\mathrm{p}_{\text {trend }} \ddagger$ & & 0.052 & 0.043 \\
\hline \multicolumn{4}{|c|}{ Attendance of Mental Health Service§, ๆ } \\
\hline Discontinue & $417(75)$ & 1.00 (reference) & 1.00 (reference) \\
\hline Regular & $139(25)$ & $1.07(0.74$ to 1.55$)$ & $0.98(0.63$ to 1.51$)$ \\
\hline \multicolumn{4}{|l|}{ Treatments§ } \\
\hline Patient psychotherapy & $124(22)$ & $1.08(0.73$ to 1.59$)$ & $1.02(0.66$ to 1.56$)$ \\
\hline Family psychoeducation & $319(57)$ & $1.18(0.84$ to 1.65$)$ & $1.08(0.76$ to 1.55$)$ \\
\hline Other supports & $103(18)$ & $1.42(0.97$ to 2.10$)$ & 1.35 (0.87 to 2.10$)$ \\
\hline \multicolumn{4}{|l|}{ Caregivers§ } \\
\hline Only mental health professionals & $44(8)$ & 1.00 (reference) & 1.00 (reference) \\
\hline Also other professionals & $512(92)$ & $1.41(0.74$ to 2.69$)$ & $1.27(0.65$ to 2.47$)$ \\
\hline
\end{tabular}

Estimates were rough and mutually adjusted for covariates listed in tables 1 and 2 .

${ }^{*} \mathrm{HR}$ (and $95 \% \mathrm{Cl}$ ) for the risk of hospital admission for mental disorders, according to Cox proportional hazard model. †Months with antipsychotic drugs available during the first year after index visit.

$\neq p$ Value for the trend in the risk of outcome as the category of the corresponding variable increases. §During the first year after index visit.

TAttendance was considered regular if the time span between two consecutive visits was 60 days or shorter, or discontinued, otherwise. 
Author affiliations

${ }^{1}$ Department of Statistics and Quantitative Methods, Laboratory of Healthcare Research and Pharmacoepidemiology, University of Milano-Bicocca, Milan, Italy

${ }^{2}$ Operative Unit of Territorial Health Services, Region Lombardia, Milan, Italy ${ }^{3}$ Department of Mental Health, Cà Granda Niguarda Hospital, Milan, Italy ${ }^{4}$ Department of Biomedical and Clinical Sciences Luigi Sacco, University of Milano, Milan, Italy

${ }^{5}$ Department of Mental Health, Lecco Hospital, Lecco, Italy

Contributors $\mathrm{AL}$ and $\mathrm{GC}$ generated the initial study idea. GC wrote the protocol and drafted the manuscript. DS and GC prepared the data set, and performed the data analysis and the sensitivity analysis. LM abstracted the data and authorised their utilisation. EM assisted in interpreting the results under clinical prospective. All authors interpreted the results.

Funding This research received no specific grant from any funding agency in the public, commercial or not-for-profit sectors.

Competing interests None declared.

Provenance and peer review Not commissioned; externally peer reviewed.

Data sharing statement No additional data are available.

Open Access This is an Open Access article distributed in accordance with the Creative Commons Attribution Non Commercial (CC BY-NC 4.0) license, which permits others to distribute, remix, adapt, build upon this work noncommercially, and license their derivative works on different terms, provided the original work is properly cited and the use is non-commercial. See: http:// creativecommons.org/licenses/by-nc/4.0/

\section{REFERENCES}

1. American Psychiatric Association. Diagnostic and Statistical Manual of Mental Disorders. 5th edn. Washington DC: American Psychiatric Association, 2013.

2. Dominguez Mde G, Viechtbauer W, Simons CJ, et al. Are psychotic psychopathology and neurocognition orthogonal? A systematic review of their associations. Psychol Bull 2009;135:157-71.

3. Jablensky A. Prevalence and incidence of schizophrenia spectrum disorders: implications for prevention. Aust N Z J Psychiatry 2000;34 (Suppl):S26-34; discussion S35-S38.

4. Saha S, Chant D, McGrath J. A systematic review of mortality in schizophrenia: is the differential mortality gap worsening over time? Arch Gen Psychiatry 2007;64:1123-31.

5. Chien WT, Yip AL. Current approaches to treatments for schizophrenia spectrum disorders, part I: an overview and medical treatments. Neuropsychiatr Dis Treat 2013;9:1311-32.

6. Bilder RM. Neurocognitive impairment in schizophrenia and how it affects treatment options. Can J Psychiatry 1997;42:255-64.

7. Bustillo J, Lauriello J, Horan W, et al. The psychosocial treatment of schizophrenia: an update. Am J Psychiatry 2001;158:163-75.

8. Carpenter WT Jr, Heinrichs DW, Wagman AM. Deficit and nondeficit forms of schizophrenia: the concept. Am J Psychiatry 1988;145:578-83.

9. Hogarty GE, Anderson CM, Reiss DJ, et al. Family psychoeducation, social skills training, and maintenance chemotherapy in the aftercare treatment of schizophrenia. I. One-year effects of a controlled study on relapse and expressed emotion. Arch Gen Psychiatry 1986;43:633-42.

10. Patterson TL, Leeuwenkamp OR. Adjunctive psychosocial therapies for the treatment of schizophrenia. Schizophr Res 2008;100:108-19.

11. Pfammatter M, Junghan UM, Brenner HD. Efficacy of psychological therapy in schizophrenia: conclusions from meta-analyses. Schizophr Bull 2006;32(Suppl 1):S64-80.

12. Rector NA, Beck AT. Cognitive behavioral therapy for schizophrenia: an empirical review. J Nerv Ment Dis 2001;189:278-87.

13. Tandon R, Targum SD, Nasrallah HA, et al. Treatment Effectiveness in Schizophrenia Consortium. Strategies for maximizing clinical effectiveness in the treatment of schizophrenia. J Psychiatr Pract 2006;12:348-63.
14. Lora A, Kohn R, Levav I, et al. Service availability and utilization and treatment gap for schizophrenic disorders: a survey in 50 low- and middle-income. Bull World Health Organ 2012;90:47-5.

15. Tansella $M$, Thornicroft $G$, Lempp $H$. Lessons from community mental health to drive implementation in health care systems for people with long-term conditions. Int J Environ Res Public Health 2014:11:4714-28

16. Lora A, Barbato A, Cerati G, et al. The mental health system in Lombardy, Italy: access to services and patterns of care. Soc Psychiatry Psychiatr Epidemiol 2012;47:447-54.

17. Conti V, Lora A, Cipriani A, et al. Persistence with pharmacological treatment in the specialist mental healthcare of patients with severe mental disorders. Eur J Clin Pharmacol 2012;68:1647-55.

18. Barbui C, Conti V, Purgato $\mathrm{M}$, et al. Use of antipsychotic drugs and mood stabilizers in women of childbearing age with schizophrenia and bipolar disorder: epidemiological survey. Epidemiol Psychiatr Sci 2013;22:355-61.

19. Charlson ME, Charlson RE, Peterson JC, et al. The Charlson comorbidity index is adapted to predict costs of chronic disease in primary care patients. J Clin Epidemiol 2008;61:1234-40.

20. Schneeweiss S. Sensitivity analysis and external adjustment for unmeasured confounders in epidemiologic database studies of therapeutics. Pharmacoepidemiol Drug Saf 2006;15:291-303.

21. Marder SR. Monitoring treatment and managing adherence in schizophrenia. J Clin Psychiatry 2013;74:e21.

22. Hui CL, Wong GH, Tang JY, et al. Predicting 1-year risk for relapse in patients who have discontinued or continued quetiapine after remission from first-episode psychosis. Schizophr Res 2013;150:297-302

23. Uchida T, Suzuki T, Sakurai H, et al. Ten year outcomes of outpatients with schizophrenia on conventional depot antipsychotics: a systematic chart review. Int Clin Psychopharmacol 2013;28: 261-6.

24. Schennach R, Obermeier M, Meyer S, et al. Predictors of relapse in the year after hospital discharge among patients with schizophrenia. Psychiatr Serv 2012;63:87-90.

25. Subotnik KL, Nuechterlein $\mathrm{KH}$, Ventura J, et al. Risperidone nonadherence and return of positive symptoms in the early course of schizophrenia. Am J Psychiatry 2011;168:286-92.

26. Leucht S, Tardy M, Komossa K, et al. Antipsychotic drugs versus placebo for relapse prevention in schizophrenia: a systematic review and meta-analysis. Lancet 2012;379:2063-71.

27. Gaebel W, Weinmann S, Sartorius N, et al. Schizophrenia practice guidelines: international survey and comparison. Br J Psychiatry 2005; 187:248-55.

28. Mulvany F, O'Callaghan E, Takei N, et al. Effect of social class at birth on risk and presentation of schizophrenia: case-control study. BMJ 2001;323:1398-401.

29. Surendra P, Singh SP, Singh V, et al. Efficacy of antidepressants in treating the negative symptoms of chronic schizophrenia: meta-analysis. Br J Psych 2010;197:174-9.

30. Corrao G, Zambon A, Nicotra F, et al. Persistence with oral and transdermal hormone replacement therapy and hospitalisation for cardiovascular outcomes. Maturitas 2007;57:315-24.

31. Corrao G, Zambon A, Conti V, et al. Menopause hormone replacement therapy and cancer risk: an Italian record linkage investigation. Ann Oncol 2008;19:150-5.

32. Corrao G, Parodi A, Zambon A, et al. Reduced discontinuation of antihypertensive treatment by two-drug combination as first step. Evidence from daily life practice. J Hypertens 2010;28 1584-90.

33. Steiner JF, Prochazka AV. The assessment of refill compliance using pharmacy records: methods, validity, and applications. $J$ Clin Epidemiol 1997;50:105-16.

34. Corrao G, Mancia G. Generating evidence from computerized healthcare utilization databases. Hypertension 2015;65:490-8.

35. Ray WA. Evaluating medication effects outside of clinical trials: new-user designs. Am J Epidemiol 2003;158:915-20.

36. Corrao G, Ghirardi A, Segafredo G, et al, BEST Investigators. User-only design for assessing drug effectiveness in clinical practice: application to bisphosphonates and secondary prevention of fracture. Pharmacoepidemiol Drug Saf 2014;23:859-67.

37. Stürmer T, Funk MJ, Poole C, et al. Nonexperimental comparative effectiveness research using linked healthcare databases. Epidemiology 2011;22:298-301. 